\title{
Sarcoma histiocítico cutáneo. Reporte de un caso
}

\author{
Katty Schnettler ${ }^{1}$, Claudia Salomone², José R Valbuena ${ }^{1}$. \\ Cutaneous histiocytic sarcoma. \\ Report of one case
}

\begin{abstract}
Histiocytic sarcoma (HS) is a rare hematologic malignancy with morphologic and immunophenotypic evidence of histiocytic differentiation. Previously known as "true histiocytic lymphoma", the tumor follows an aggressive clinical course. Involvement of lymph nodes, skin, and extranodal sites has been described. Among the latter, approximately 29 cases involving the skin have been reported. On pathology, the lesion shows a diffuse proliferation of large epitheloid histiocytes with marked pleomorphism. We report a 78 year-old male who presented with a nodular lesion in the forehead, involving the skin and soft tissue. The pathological diagnosis was a HS. The patient was lost from follow up (Rev Méd Chile 2009; 137: 547-51).
\end{abstract}

(Key words: Hematologic malignancies; Histiocytic sarcoma; Neoplasm metastasis)

Recibido el 10 de abril, 2008. Aceptado el 10 de noviembre, 2008.

Departamentos de Anatomía Patológica ${ }^{1}$ y Dermatología ${ }^{2}$, Facultad de Medicina, Pontificia Universidad Católica de Chile, Santiago de Chile.

$E^{1}$ sarcoma histiocítico (SH) es una rara neoplasia maligna, que se presenta frecuentemente con enfermedad clínicamente avanzada y curso agresivo $^{1-6}$. Ha sido descrito en ganglios linfáticos, piel y otros sitios extranodales ${ }^{1-5}$. El $\mathrm{SH}$ en el pasado era un diagnóstico relativamente frecuente, sin embargo, el estudio inmunohistoquímico demuestra que la mayoría de los casos descritos representan en realidad otros tipos de neoplasias, tales como melanoma, linfoma B difuso de células grandes (LBDCG) o linfoma anaplásico de células grandes (LACG) ${ }^{9,15,16}$. Por lo tanto, muchas inconsistencias en su terminología y criterios diagnósti-

Correspondencia a: Dr. José R. Valbuena. Departamento de Anatomía Patológica, Facultad de Medicina, Pontificia Universidad Católica de Chile. Lira 85, 4으 Piso. Santiago, Chile. Teléfono: +562 3543252. Fax: +56 26395101 .

E mail: jvalbuen@med.puc.cl cos han complicado su reconocimiento y caracterización. Describimos un caso de SH cutáneo en nuestro país, con estudio inmunohistoquímico y discutimos lo comunicado hasta el momento en la literatura.

\section{CASO CLÍNICO}

Hombre de 78 años de edad, sin antecedentes mórbidos, consultó por aumento de volumen en la frente, de 2 meses de evolución, único, sin sintomatología asociada. Al examen físico presentaba un tumor firme, elástico, no adherido a planos profundos, de $1,7 \times 0,6 \mathrm{~cm}$, con algunas pápulas eritematosas levemente translúcidas en la superficie y múltiples telangiectasias (Figura 1). Se extrajo la lesión y se envió a anatomía patológica con diagnóstico clínico de carcinoma basocelular sobre quiste epidérmico. Al momento del control clínico, una semana después, presentó otro tumor

C A S O S

CLÍNICOS 
— Rev Méd Chile 2009; 137: 547-551

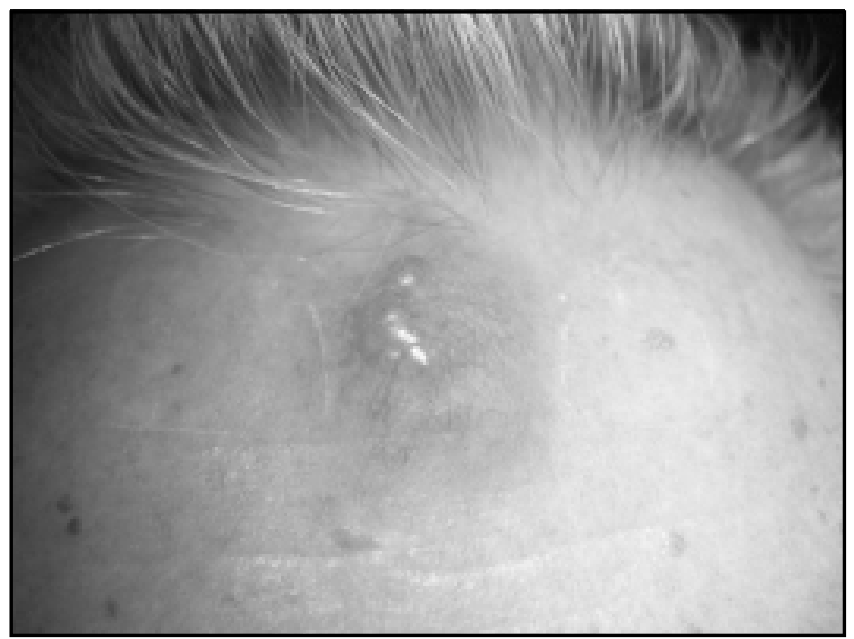

Figura 1. Paciente de 78 años con tumor en la frente de 1,7 x 0,6 cm.

más pequeño en la región supraciliar derecha, de similares características al primero. El examen anatomopatológico del tumor frontal mostró una neoplasia compatible con SH. Estudios de imágenes (tomografía computada de cabeza y cuello) mostraron múltiples lesiones nodulares en tejido celular subcutáneo en ambas regiones temporales, así como adenopatías submandibulares y yugulodigástricas. También se observaron nódulos en ambas glándulas parótidas. Estudios de laboratorio incluyeron hemograma que mostró los siguientes valores: hemoglobina, 16,0 $\mathrm{gr} / \mathrm{dL}$ (valores de referencia (VR), 13,5-17,5); hematocrito, 45,8\% (VR, 41-53); leucocitos, 5,0 x $10^{3} / \mathrm{mm}^{3}$ (VR, 4,511) y plaquetas, $200 \times 10^{3} / \mathrm{mm}^{3}$ (VR, 140-400). El recuento leucocitos fue: neutrófilos, $53 \%$; eosinófilos, $4 \%$; monocitos, $18 \%$ y linfocitos, $25 \%$. Otros exámenes de laboratorio (perfil bioquímico y pruebas hepáticas) estuvieron dentro de rangos normales. El paciente fue tratado en otra institución y desafortunadamente se perdió para seguimiento clínico.

\section{HALIAZGOS HISTOLÓgICOS}

Microscópicamente, el tumor frontal mostró una proliferación celular atípica, difusa, en dermis superficial y profunda, no epidermotrópica y con zona de Grenz. Constituida por células medianas a grandes, de aspecto epiteloideo y pleomorfismo variable, que incluye formas multinucleadas (Figuras $2 \mathrm{~A}$ a $2 \mathrm{C}$ ). Se observó además un componente linfocitario prominente, numerosas mitosis y cuerpos apoptóticos.

Inmunohistoquímicamente, las células neoplásicas mostraron reacción positiva para CD43, CD45, CD4 (parcial), CD30 (difuso), CD68, CD163, mieloperoxidasa, lizosima y proteína S100 (Figuras 2D a 2F); y reacción negativa para TdT, CD34, CD117, CD3, CD20, HMB45 y citoqueratinas totales.

Mielograma y biopsia de médula ósea mostraron una hipoplasia medular con cambios displásicos leves. Estudio de clonalidad mediante técnica de reacción de polimerasa en cadena, mostró un reordenamiento policlonal de linfocitos $\mathrm{T}$ y $\mathrm{B}$.

\section{DisCUSIÓN}

El SH, anteriormente designado como linfoma histiocítico verdadero, es una neoplasia maligna rara de origen hematopoyético que muestra evidencias morfológicas e inmunofenotípicas de diferenciación histiocítica ${ }^{1,4,5}$. El SH ha sido reportado en ganglios linfáticos, piel y sitios extranodales, principalmente tracto gastrointestinal, presentándose frecuentemente con enfermedad clínicamente avanzada y curso agresivo ${ }^{1-5}$. En la piel se han reportado aproximadamente 29 casos, como casos aislados y en pequeñas series ${ }^{2,3,7-14}$. 


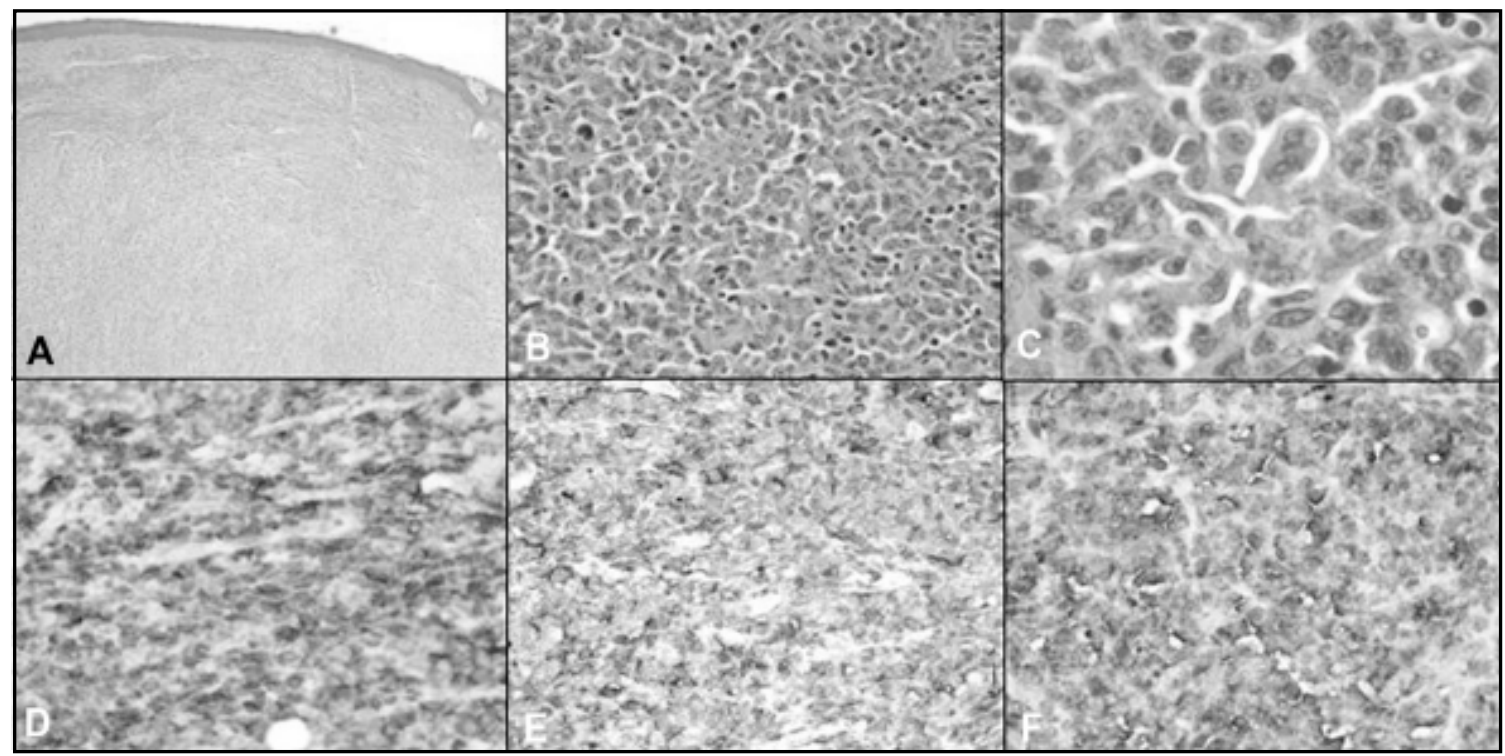

Figura 2. Sarcoma histiocítico cutáneo. Proliferación celular atípica, no epidermotrópica, constituida por células medianas a grandes, con abundante citoplasma eosinófilo y grado variable de pleomorfismo, con células multinucleadas y un componente linfocitario, hematoxilina eosina, 40x (A) y 400x (B) y 1000x, aceite de inmersión (C). Tinciones inmunohistoquímicas para CD45 (D), Lisozima (E) y CD163 (F), 400x.

Previo al desarrollo y amplio uso de técnicas inmunohistoquímicas, el $\mathrm{SH}$ era un diagnóstico relativamente frecuente. Muchas inconsistencias en la terminología y criterios diagnósticos de estas lesiones históricamente han complicado su reconocimiento y caracterización. De hecho, la mayoría de los casos descritos en el pasado representan ejemplos de linfoma no Hodgkin (LNH), predominantemente LBDCG y LACG9,15,16.

El SH se presenta en un amplio rango de edades. Sin embargo, la mayoría de los casos ocurre en adultos (46 años en promedio). Los pacientes pueden presentarse con una masa solitaria, y generalmente con síntomas sistémicos, tales como fiebre y baja de peso. Las manifestaciones cutáneas van desde un eritema aparentemente benigno a lesiones solitarias y numerosos tumores en tronco y extremidades. Pacientes con lesiones intestinales pueden manifestarse con obstrucción intestinal. La hepatoesplenomegalia es relativamente común. El hueso puede mostrar lesiones líticas, y por definición no debe haber compromiso medular 5 .

El diagnóstico de $\mathrm{SH}$ se basa en la verificación de diferenciación histiocítica, con marcadores in- munohistoquímicos para CD68, CD163 y lizosima, y en la exclusión de otras neoplasias de células grandes, poco diferenciadas, tales como linfoma, carcinoma o melanoma.

Dentro de las neoplasias de origen no hematopoyético, el melanoma es la neoplasia cutánea no epitelial más frecuente. En fase tumoral puede mostrar una histología pleomórfica e indiferenciada. Al igual que el $\mathrm{SH}$, la mayoría de los melanomas expresan inmunorreactividad para la proteína S-100, pero no muestran expresión de antígenos de diferenciación hematopoyética 0 monocítica, tales como CD45 y CD163, respectivamente $^{1,6}$. Neoplasias de origen epitelial expresan en grado variable diferentes tipos de queratinas, de utilidad para excluir este tipo de neoplasias.

Los linfomas cutáneos más frecuentemente incluidos en el diagnóstico diferencial es el LACG, primario o secundario, linfoma/leucemia linfoblástica (LB) y LBDCG. El LACG expresa antígenos de linfocitos $\mathrm{T}$, muestra expresión característica para CD30 (membrana y paranuclear) y en algunos casos para ALK-1. Por su parte, el LBDCG muestra marcadores de diferenciación de estirpe $\mathrm{B}$, como la positividad variable para BCL2, BCL6, CD20 y 
CD79. El LB, además de mostrar diferenciación de células $\mathrm{T}$ o $\mathrm{B}$, expresan marcadores de inmadurez tales como TdT y CD10, respectivamente ${ }^{1,5}$. Por último, estudios complementarios de clonalidad para linfocitos T y B ayudan a establecer un diagnóstico definitivo.

El diagnóstico diferencial incluye además síndromes proliferativos agudos o crónicos con diferenciación mielomonocítica, extramedulares, como es el caso del sarcoma granulocítico (SG) o tumor mieloide extramedular (TME).

El SG o TME generalmente se presenta en pacientes con leucemias mieloides agudas o crónicas en algún momento de su evolución $n^{5,17}$, y en casos raros como su presentación inicial ${ }^{18}$. Dentro del grupo de leucemias mieloides que frecuentemente pueden presentarse como neoplasias extramedulares se encuentran aquellas con diferenciación monocítica/histiocítica. De hecho la distinción entre SH y TME con diferenciación monocítica es difícil, ya que son inmunohistoquímicamente indistinguibles. Ambas pueden comprometer sitios inusuales, tales como la piel, y tienen un curso clínico agresivo ${ }^{11}$. En muchos casos la diferenciación resulta algo arbitraria, y pueden representar diferente espectro de un mismo trastorno.

Aun así, como en nuestro caso, existen ciertas diferencias morfológicas e inmunofenotípicas entre SH y TME. Morfológicamente, el TME tiende a ser una proliferación celular más o menos homogénea, con menos pleomorfismo que el $\mathrm{SH}$ y puede contener elementos granulocíticos normales. Se subdivide en TME bien diferenciado (mezcla de elementos maduros e inmaduros), poco diferenciado (solamente promielocitos/promonocitos) e indiferenciados (de tipo blástico) ${ }^{19}$. Por lo general, los TME muestran positividad variable para $\mathrm{CD} 34$ o $\mathrm{CD} 117^{18}$, mientras que usualmente el $\mathrm{SH}$ es negativo para ambos ${ }^{1}$.

Otras proliferaciones histiocíticas poco comunes con compromiso cutáneo, corresponden a reticulohistiocitoma cutáneo (RC) y enfermedad de Rosai-Dorfmann (ERD) ${ }^{20,21}$. La reticulohistiocitosis tiene dos formas de presentación, solitaria y multicéntrica, usualmente ocurre en adultos jóvenes, pero tiene un amplio rango de edades. El RC se presenta como una lesión cutánea pequeña (menos de $1 \mathrm{~cm}$ ), superficial, que ocasionalmente se extiende al tejido subcutáneo. El tumor típicamente está compuesto por histiocitos epiteloideos grandes, con abundante citoplasma eosinófilo, entremezclado con linfocitos y a veces granulocitos. La atipía nuclear es leve y las mitosis son raras. El patrón de expresión de marcadores histiocíticos es casi indistinguible del SH. Sin embargo, la consistente detección de CD45 en SH, comparado con su expresión débil y variable en $\mathrm{RC}$, podría ser una diferencia útil en la separación de estas entidades ${ }^{20}$.

La ERD cutánea es rara, generalmente se presenta una lesión cutánea asintomática, predominantemente en pacientes asiáticos. Se reconocen histiocitos poligonales, un infiltrado inflamatorio mixto con numerosas células plasmáticas y fibrosis. La tinción inmunohistoquímica para proteína S-100 es positiva. La diferencia con el SH radica en el grado de atipia y la ausencia de emperipolesis, en este último ${ }^{21}$.

Finalmente, las mastocitosis sistémicas o cutáneas y las histiocitosis de células de Langerhans (HCL) usualmente comprometen la piel. El mastocitoma cutáneo (MC) clínicamente puede presentarse como un tumor solitario, pero a diferencia del $\mathrm{SH}$, está compuesto de nidos de mastocitos dérmicos, con abundante citoplasma eosinófilo, con formas fusiformes, que pueden extenderse al tejido subcutáneo 22 . El MC muestra positividad para CD25, CD117 y triptasa, lo cual lo diferencia de un SH. La HCL, usualmente es una enfermedad multisistémica y de comportamiento agresivo. Histológicamente, está compuesta por una proliferación de células atípicas con núcleos irregulares con ranuras nucleares, con o sin atipía, y presenta acentuada eosinofilia tisular; inmunohistoquímicamente expresa proteína S-100 y CD1a; y ultraestructuralmente contienen en su citoplasma gránulos de Birbeck ${ }^{5}$.

Desde el punto de vista terapéutico, dado la rareza de esta entidad, actualmente no hay terapias estandarizadas. Sin embargo, esquemas de quimioterapia con agentes múltiples similar al tratamiento de LNH y el uso de talidomida parecen ser las modalidades terapéuticas más utilizadas ${ }^{1,23,24}$.

En conclusión, el $\mathrm{SH}$ es un diagnóstico de exclusión. Se requiere una estricta correlación de hallazgos clínicos y patológicos, y un estudio inmunohistoquímico extenso para un adecuado diagnóstico. Este es el primer caso estudiado ampliamente y reportado en la literatura médica local. 


\section{REFERENCIAS}

1. Hornick JL, JAFFe ES, Fletcher CD. Extranodal histiocytic sarcoma. Am J Surg Pathol 2004; 28: 1133-44.

2. Copie-Bergman C, Wotherspoon AC, Norton AJ, Diss TC, ISAACSON PG. True histiocytic lymphoma: a morphologic, inmunohistochenical, and molecular genetic study of 13 cases. Am J Surg Pathol 1998; 22: 1386-92.

3. Pileri SA, Grogan TM, Harris NL, Banks P, Campo E, CHAN JKC ET AL. Tumours of histiocytic and accessory dendritic cells: an immunohistochemical approach to classification from the International Lymphoma Study Group based on 61 cases. Histopathology 2002; 41: 1-29.

4. Warnke RA, Weiss LM, Chan JKC, Cleary ML, Dorfman RF. Atlas of Tumor Pathology: Tumors of the Lymph Nodes and Spleen, third series, fascicle 14. Washington, DC: Armed Forces Institute of Pathology; 1995.

5. Jaffe ES, Harris NL, Stein H, Vardiman JW. (Eds.): World Health Organization Classification of $\mathrm{Tu}$ mours. Pathology and Genetics of Tumours of Haematopoietic and Lymphoid Tissues. IARC Press: Lyon 2001.

6. Vos JA, Abbondanzo SL, Barekman CL, Andriko JW, MietTinen M, Aguilera NS. Histiocytic sarcoma: a study of five cases including the histocyte marker CD163. Mod Pathol 2005; 18: 693-704.

7. Willemze R, Ruiter DJ, Van Vloten WA, Mejer CJ. Reticulum cell sarcomas (large cell lymphomas) presenting in the skin. High frequency of true histiocytic lymphoma. Cancer 1982; 50: 1367-79.

8. Van Der Valk P, Meijer CJ, Wiluemze R, Van Oosterom AT, SpaAnder PJ, Te Velde J. Histiocytic sarcoma (true histiocytic lymphoma): a clinicopathological study of 20 cases. Histopathology 1984; 8: 105-123.

9. Arai E, Su WP, Roche PC, L CY. Cutaneous histiocytic malignancy. Immunohistochemical re-examination of cases previously diagnosed as cutaneous "histiocytic lymphoma" and "malignant histiocytosis". J Cutan Pathol 1993; 20: 115-20.

10. Osborne BM, Mackav B. True histiocytic lymphoma with multiple skin nodules. Ultrastruct Pathol 1994; 18: 241-6.

11. Lauritzen AF, Delsol G, Hansen NE, Horn T, Ersbølu J, Hou-JeNSEN K ET AL. Histiocytic sarcomas and monoblastic leukemias. A clinical, histologic, and immunophenotypical study. Am J Clin Pathol 1994; 102: 45-54.

12. Boisseau-Garsaud AM, Vergier B, Beylot-Barry M, Nastasel-Menini F, Dubus P, De Mascarel A et al.
Histiocytic sarcoma that mimics benign histiocytosis. J Cutan Pathol 1996; 23: 275-82.

13. De Paz R, Canales MA, Morado M, HernándezNAVARRo F. Sarcoma histiocítico: un nuevo caso de presentación clínica atípica. Med Clin (Barc) 2005; 124: 798-9.

14. Frater JL, Kung CW, Obadiah JM, Gardner LJ, Grosso LE, RESH B ET AL. Histiocytic sarcoma with secondary involvement of the skin and expression of CD1a: evidence of indeterminate cell differentiation? J Cutan Pathol 2006; 33: 437-42.

15. Van Der Valk P, Van Oostveen JW, Stel HV, Van Der Kwast TH, Melief CJ, MejJer CJ. Phenotypic and genotypic analysis of large-cell lymphomas, formerly classified as true histiocytic lymphoma: identification of an unusual group of tumors. Leuk Res 1990; 14: $337-46$

16. Wilson MS, Weiss LM, GatTer KC, Mason DY, Dorfman RF, WARNKE RA. Malignant histiocytosis: a reassessment of cases previously reported in 1975 bases on paraffin section immunophenotyping studies. Cancer 1990; 66: 530-6.

17. Cho-Vega JH, Medeiros LF, Prieto VG, Vega F. Leukemia cutis. Am J Clin Pathol 2008: 129: 130-42.

18. Valbuena JR, Admirand JH, Lin P, Medeiros LJ. Myeloid sarcoma involving the testis. Am J Clin Pathol 2005; 124: 445-52.

19. TRaWeEk ST, Arber DA, Rappaport $H$, Brynes RK. Extramedullary myeloid cell tumors. An immunohistochemical and morphologic study of 28 cases. Am J Surg Pathol 1993; 17: 1011-9.

20. Miettinem M, Fetsch JF. Reticulohistiocytoma (solitary ephiteloid histiocytoma) a clinicopathologic and immunohistochemical study of 44 cases. Am J Surg Pathol 2006; 30: 521-8.

21. Kong Y, Kong J, Shi D, Lu H, Zhu X, Wang J et al. Cutaneous Rosai-Dorfman Disease. A clinical and histopathologic study of 25 cases in China. Am J Surg Pathol 2007; 31: 341-50.

22. Wolff K, Komar M, Petzelbauer P. Clinical and histopathological aspects of cutaneous mastocytosis. Leuk Res 2001; 25: 519-28.

23. Dale JH, Leblond P, Decouvelaere A, Yakoub-Agha I, Preudhomme C, NelKen B et al. Efficacy of thalidomide in a child with histiocytic sarcoma following allogeneic bone marrow transplantation for T-ALL. Leukemia 2003; 17: 2056-7.

24. Abid MH, Tove I, Ibrahim RB, Maria D, Peres E. Thalidomide for the treatment of histiocytic sarcoma after hematopoietic stem cell transplant. Am J Hematol 2007; 82: 932-3. 